\title{
The common marmoset (Callithrix jacchus): a relevant preclinical model of human (auto)immune-mediated inflammatory disease of the brain
}

\author{
Bert A. 't Hart, Jordon Dunham, S. Anwar Jagessar, and Yolanda S. Kap \\ Department Immunobiology, Biomedical Primate Research Centre, Rijswijk, the Netherlands \\ Correspondence to: Bert A. t'Hart (hart@bprc.nl)
}

Received: 5 November 2015 - Revised: 7 January 2016 - Accepted: 11 January 2016 - Published: 12 February 2016

\begin{abstract}
The increasing prevalence of chronic autoimmune-mediated inflammatory disorders (AIMIDs) in aging human populations creates a high unmet need for safe and effective medications. However, thus far the translation of pathogenic concepts developed in animal models into effective treatments for the patient has been notoriously difficult. The main reason is that currently used mouse-based animal models for the pipeline selection of promising new treatments were insufficiently predictive for clinical success. Regarding the high immunological similarity between human and non-human primates (NHPs), AIMID models in NHPs can help to bridge the translational gap between rodent and man. Here we will review the preclinical relevance of the experimental autoimmune encephalomyelitis (EAE) model in common marmosets (Callithrix jacchus), a small-bodied neotropical primate. EAE is a generic AIMID model projected on the human autoimmune neuro-inflammatory disease multiple sclerosis (MS).
\end{abstract}

\section{Introduction}

Aging Western societies are facing an increasing prevalence of chronic (auto)immune-mediated inflammatory diseases (AIMIDs) for which an effective treatment is either lacking or associated with detrimental side effects. This challenging situation creates a need for translationally relevant animal models in which the (immuno)pathogenesis of AIMID can be investigated and in which safe and effective treatments can be developed. At the same time there has been an impressive development in the field of biotechnology, which now enables the transformation of biological molecules into highly specific therapeutic bullets, monoclonal antibodies (mAbs) for example, with which pathogenic cells and molecules can be eliminated. However, although several biological drugs are now successfully used in the clinic, there is a long list of promising candidates which failed to reproduce positive effects observed in animal models when they were tested in patients. Furthermore, several clinically approved therapeutics are plagued with detrimental side effects.
One of the recognized causes for this frustrating situation is the poor predictive validity for safety and efficacy of the animal models used in preclinical research. By far the largest proportion of preclinical research in AIMID is based on a limited number of inbred and specific pathogenfree (SPF)-bred rodent strains. While rodent models are very useful in terms of reliability and reproducibility of the models, relatively low costs, the plethora of available reagents for research, and the availability of genetically modified substrains, the shortcomings of laboratory rodents as preclinical AIMID models are a clear handicap. Due to the lack of genetic diversity and exposure to environmental pathogens, the immune system of inbred SPF mice is immature and poorly comparable to the robust pathogen-educated human immune system (Davis, 2008).

We postulate here that the translational gap between disease models in mice and the corresponding human disease can be bridged by using non-human primate (NHP) disease models. The focus of this publication will be on experimental autoimmune encephalomyelitis (EAE), which models autoimmune pathogenic mechanisms of the human neuroinflammatory disease multiple sclerosis (MS) and has been 
well established in common marmosets (Callithrix jacchus)

(Vierboom et al., 2010; 't Hart et al., 2004a).

\section{Pros and cons of the marmoset as a model of human autoimmune disease}

There are some challenging limitations to usage of the marmoset for immunological research:

1. The small body size ( $\pm 400 \mathrm{~g}$ at adult age) precludes the sampling of large blood volumes. As a rule of thumb, the maximum volume that can be collected per month without harming the well-being of the animals equals $1 \%$ of the bodyweight.

2. As the marmoset is not an established model for immunological research, reagents are scarce. In the past 15 years we have compiled a useful set of reagents and methods for immunological research in the marmoset (Jagessar et al., 2013a).

3. The relatively long time (1.5-2 years) to reach sexual maturity compared to rodent species - together with the special housing, handling, and dietary needs of the marmose - increase costs of research substantially.

4. Facility requirements limit the ability of many research institutes to perform research using the marmoset. Additionally, animal handlers require specialized training.

5. Regulatory restrictions due to the NHP status of the marmoset, and welfare considerations, are a serious limiting factor in research performed. These limitations can be overcome by careful study design and daily veterinary care.

There are also several clear advantages of this model:

1. Despite the small body size (Jagessar et al., 2013a) it is possible to obtain meaningful longitudinal information about the monkey's immune status by using micromethods detailed elsewhere (Jagessar et al., 2013).

2. The marmoset is a friendly animal that adapts well to experimental handlings, without the need of sedation, and responds well to training procedures designed for testing cognitive function.

3. Marmosets usually give birth to two or three nonidentical siblings. During the in utero development the placental blood stream of twin siblings is fused, which implies that they have a chimeric bone marrow (Haig, 1999; Picus et al., 1985). The ensuing stable allotolerance creates the possibility to adoptively transfer immune cells between fraternal siblings. Moreover, as the immune systems of such twins have been educated in the same thymic environment, they are highly similar. This chimeric bone marrow status is highly advantageous for placebo-controlled studies designed to test potential therapeutics as the fraternal sibling can be used as an optimal control. Such a paired approach reduces the number of monkeys needed for research.

4. Marmosets in captivity can reach a high age, above 20 years. It is unknown, however, whether the immune system ages in the same way as the human immune system. Importantly, marmosets are naturally infected with similar viruses to humans, including those related to viruses implicated in the aging of the human immune system, namely the $\beta$-herpesvirus CMV (cytomegalovirus) and the $\gamma$-herpesvirus EBV (EpsteinBarr Virus) (Nigida et al., 1979; Cho et al., 2001).

5. Just like humans, marmosets held in captive colonies under conventional conditions have a robust pathogeneducated immune system, which comprises specificities engaged in the control of chronic latent herpesvirus infection. We will discuss in this publication that the marmoset versions of CMV and EBV have a central role in the pathogenesis of MS-like disease in marmosets.

6. The need to formulate the immunizing antigen with strong immune stimulators, such as the complete Freund's adjuvant (CFA), for reproducible induction of AIMID in laboratory animals raises concerns. CFA is notorious for the adverse effects, which cause serious discomfort to the animals (Billiau and Matthys, 2001). Moreover, CFA causes skewing of the $T$ cell response to the immunizing (auto)antigen into a pro-inflammatory profile that is dominated by Th1 and Th17 cells. The thus far-reaching disappointing effects in MS therapies targeting these subsets indicate that CFA-based EAE models may be less representative for MS. We recently discovered that full-blown clinical EAE in marmosets can be induced with myelin antigen formulated with the much less detrimental incomplete Freund's adjuvant (IFA) (Jagessar et al., 2010, 2015). IFA lacks the mycobacteria that cause the adverse effects.

\section{Multiple sclerosis (MS)}

MS is an inflammatory/demyelinating disease of the human central nervous system (CNS). In the vast majority of patients $( \pm 85 \%)$ the disease follows initially a relapsingremitting course characterized by alternating episodes of disease activity and recovery. In about $50 \%$ of these relapsingremitting MS (RRMS) cases and after a variable period of time the disease worsens progressively and remissions are less frequently observed, i.e., secondary progressive disease (SPMS). In $15 \%$ of the patients the disease is progressive from the onset, i.e., primary progressive disease (PPMS) (Dendrou et al., 2015). The cause of MS is unknown, but, 
once established, disease evolution is driven by the combined activity of autoreactive $T$ and $B$ cells specific for components of the myelin sheaths that wrap around axons in the CNS white matter. Genome-wide association studies and the beneficial effect of immunomodulatory therapies support a central pathogenic role of the immune system. However, immunemodulatory or immune-suppressive therapies mainly have an effect in the relapsing-remitting phase of MS; it is therefore believed that the immune system exerts its main pathogenic functions during this phase and is less important for the progressive phase.

It is generally believed that autoimmunity in MS is caused by the interaction of genetic and environmental risk factors. Low vitamin D, EBV infection, and smoking are all widely considered environmental risk factors associated with MS (Ascherio and Munger, 2007a, b). Genome-wide association studies have shown that more than 100 genes contribute to the genetic susceptibility (Sawcer et al., 2011). The strongest genetic influence is exerted by the major histocompatibility complex (MHC) class II region. The individual contribution of the other genes, which all encode an immunological function, is more modest. MHC II molecules are expressed on antigen-presenting cells (APCs) and present antigens to CD4 $+T$ cells. Although this association is suggestive for a core pathogenic role of CD4 $+T$ cells in MS, therapies targeting this cell type lack convincing efficacy in RRMS (see below).

Despite decades of intensive research a viral or bacterial trigger, which withstands Koch's postulates, has not been found. There is strong evidence for a pathogenic role of EBV infection, but the discrepancy between the high prevalence of EBV infection in the human population $(>90 \%)$ and the low incidence of MS $( \pm 0.1 \%)$ is unexplained (Ascherio et al., 2012). The risk for MS increases 2-3-fold with development of infectious mononucleosis compared to EBVseropositive people, and an increased IgG1 titer against EBV proteins EBNA-1 is predictive of disease exacerbation (Ascherio and Munger, 2015). MS development is virtually not seen in EBV-seronegative individuals (Pakppor et al., 2013), providing further evidence of a link between EBV and MS. Critical to this discussion, and as previously mentioned, is the fact that marmosets are naturally infected with an EBV homolog (CalHV3). We will discuss in following paragraphs data obtained in the marmoset EAE model, which may provide a mechanistic explanation of the enigmatic association between MS and EBV infection.

The uncertainty about an external cause of MS has led others (Stys et al., 2012) and us ('t Hart er al., 2009) to propose that the cause may be inside the CNS. Our "responseto-injury" concept of MS has been inspired by the old "primary lesion hypothesis" proposed by Wilkin (1989). According to this hypothesis, "autoimmunity is a genetically predisposed hyper-response to self antigens released by tissue injury (= the primary lesion) due to an antecedent pathogenic event". We will explain in the following sections that unique aspects of the marmoset model provide the opportunity for testing hypotheses on the enigmatic trigger of the primary lesion and the ensuing autoimmune reactions.

\section{Modeling the cause of the primary lesion}

It is generally assumed that the pathogenic event that elicits MS predates the presentation of neurological deficits for many years. This obviously complicates the identification of that elusive event. However, in a recent publication remarkable observations were reported that may provide useful insights ('t Hart, 2015): (1) while MS has always been rare in Japan, the annual incidence curve has dramatically increased since World War II. This increment has been attributed to the introduction of the Western diet (Yamamura and Miyake, 2013). (2) MS seems to affect only humans. Even our closest living relatives, chimpanzees and bonobos, are not affected by the disease despite the high genetic similarity and the fact that they are naturally affected with similar pathogens to humans. This suggests that MS is due to a pathogenic condition that is only present in humans.

Analysis of the earliest pathological changes in the myelin of MS patients (called pre-active lesions; van der Valk and Amor, 2009) revealed aggregates of microglia cells around abnormal axons, called microglia nodules (Singh et al., 2013). At the electron microscopic level demyelination seems to start with dissociation of the inner myelin lamellae from the axon (Rodriguez and Scheithauer, 1994). This has led to the concept that the primary MS lesion may start as instability of axon-myelin units (Stys et al., 2012). One of the consequences of the dissociation of myelin from axons is that the trophic support of axons with essential monocarboxylate nutrients (lactate, pyruvate) is disturbed. This inevitably results in the degeneration of axon and neuron (Saab et al., 2013; Simons et al., 2014).

Experiments in mice have shown that the interaction of myelin-associated glycoprotein (MAG) with gangliosides on the axon surface is essential for the stability of axonmyelin units (Schnaar, 2010; Schnaar et al., 2014). MAG is a sialic-acid-binding lectin (Siglec-4) with high specificity for N-acetylneuraminic acid (Neu5Ac) (Collins et al., 1997). $\mathrm{Neu} 5 \mathrm{Ac}$ is the substrate for another prevalent sialic acid, Nglycolylneuraminic acid (Neu5Gc). Interestingly, expression of the Neu5Ac into Neu5Gc-converting enzyme, CMP-Nacetylneuraminic acid hydroxylase (CMAH), is suppressed in the CNS, suggesting that expression of Neu5Gc in the brain and spinal cord is detrimental for homeostasis (Davies and Varki, 2013).

Varki et al. reported a genetic deficiency of the enzyme CMAH in humans that is not present in hominoid apes (Varki, 2001). Due to this deficiency humans are unable to synthesize Neu5GC anywhere in their whole body, while chimpanzees produce it in all body cells except the CNS. Neu5GC is, however, present in abundance in the red meat of 
lifestock (cow, pig, goat), which is consumed in large quantities via the Western diet, but it is absent in fish, poultry and vegetables (Arnon et al., 1996; Wang, 2009). Intriguingly, the steep increase of MS prevalence in Japan after World War II has been attributed to the replacement of the Neu5GCpoor traditional Japanese food by the Neu5GC-rich Western diet (Yamamura and Miyake, 2013). Experiments in CMAHdeficient mice have shown that dietary Neu5Gc is incorporated in the gut flora and in the glycocalyx of various body tissues (Taylor et al., 2010). The dietary modified gut flora induces the production of heterophilic antibodies, which can bind Neu5Gc incorporated in tissue glycocalyx and in this way interfere with cellular interactions and communication (Taylor et al., 2010; Samraj et al., 2014).

The questions whether dietary Neu5Gc is incorporated also in the gangliosides of the CNS and whether peripheral anti-Neu5Gc antibodies gain access to the CNS and bind thus-modified gangliosides have not been resolved. Interestingly, the common marmoset has the same genetic CMAH deficiency as humans (Springer et al., 2014) and therefore provides an exquisite model to examine this unexplored ethiogenic mechanism in further detail.

\section{Modeling response to myelin injury}

Studies in rats where CNS white-matter injury was inflicted via a cryolesion have shown that myelin debris can be found within CNS-draining lymphoid organs (cervical and lumbar lymph nodes and the spleen) (Philips et al., 1997). Similar observations have been reported for MS (Fabriek et al., 2005) and for EAE-affected mice (van Zwam et al., 2009a) and marmosets (de Vos et al., 2002). Moreover, surgical removal of cervical and lumbar lymph nodes, which drain the spinal cord, impairs chronic relapsing EAE development in Biozzi ABH mice (van Zwam et al., 2009b). These finding support the concept that (under certain conditions) myelin released from injured CNS can be a trigger of encephalitogenic immune reactions.

We have analyzed the response of the pathogen-educated immune system of marmosets to CNS myelin from an MS patient in great detail. Marmosets immunized with MS myelin formulated with CFA developed a chronic neurological disease that resembles MS in clinical and neuropathological presentation ('t Hart et al., 1998). Just like in MS, we observed with T2-weighted MRI formation of focal hyperintense regions, which at histological examination represented focal areas of primary demyelination, characterized by variable degrees of inflammation, axonal pathology, astrogliosis, and even remyelination (Fig. 1). In later studies others and we observed that MS-like lesions were also present in the cortical grey matter, a phenomenon usually not observed in rodent EAE models (Pomeroy et al., 2008; Kap et al., 2011b). Indeed, the lack of cortical grey-matter pathology observed in the MS patient is a major pitfall of rodent EAE mod- els. Currently research is being undertaken for more in-depth pathological characterization of the marmoset EAE model. Preliminary results indicate that, in addition to grey-matter pathology, many of the desired pathological features of MS, not reflected in rodent EAE, are replicated in the marmoset EAE models (Dunham et al., 2016).

\section{Brain lesions visualized with MRI}

The anatomy of the marmoset brain, including the grey / white-matter ratio, resembles the human brain. This is especially important for studies on diseases associated with white- and grey-matter pathology. Magnetic resonance imaging is the imaging technique of choice for the visualization of MS pathology in the brain and spinal cord and is an accepted primary outcome measure in clinical trials of new therapies (Barkhof et al., 2012). The most commonly applied T2-weighted MRI technique, which we also used in our first studies, is highly sensitive to altered tissue distribution of water, vasogenic edema for example (Fig. 1a). Figure 1b shows usage of the same technique for longitudinal imaging of brain lesion development. In later years more specific imaging techniques were developed for scanners with higher field strength, such as the 4.7T lab animal scanner at the Imaging Center in Utrecht, the Netherlands. Figure 1c shows techniques used for a quantitative description of the in vivo detectable brain pathology developing in the marmoset EAE model ('t Hart et al., 2004b). Quantitative MRI techniques are ideal for determining the effect of a new therapeutic $\mathrm{mAb}$ on ongoing disease ('t Hart et al., 2006).

\section{Modeling the $T$ cell response to injury}

The high response of the pathogen-educated immune system of marmosets to CNS myelin and the multi-factorial immune reactions that underlie the neurological problems have been examined in great detail ('t Hart et al., 2015). Myelin is a complex tissue composed of (glyco)lipids, lipoproteins, proteolipids, and (glyco)proteins. We could show by immunization of Biozzi ABH mice (Smith et al., 2005) and marmosets (Jagessar et al., 2008) with myelin from mice lacking myelin oligodendrocyte glycoprotein (MOG) that this quantitatively minor constitutent $(<1 \%$ of the protein fraction) is essential for the evolution of chronic EAE.

MOG is only present in the CNS, where it is expressed as a homodimer on the surface of oligodendrocytes and myelin sheaths. The likely natural function of MOG is maintenance of an anti-inflammatory brain milieu. This role depends on the interaction of the fucosylated glycan residue, which is linked to the asparagine residue at position 31 of the extracellular domain, with the C-type lectin receptor (CLR) DC-SIGN. DC-SIGN is surface-expressed on resident APCs (microglia) and APCs within the CNS draining cervical and lumbar lymph nodes (Garcia-Vallejo et al., 2014). Thus, as 

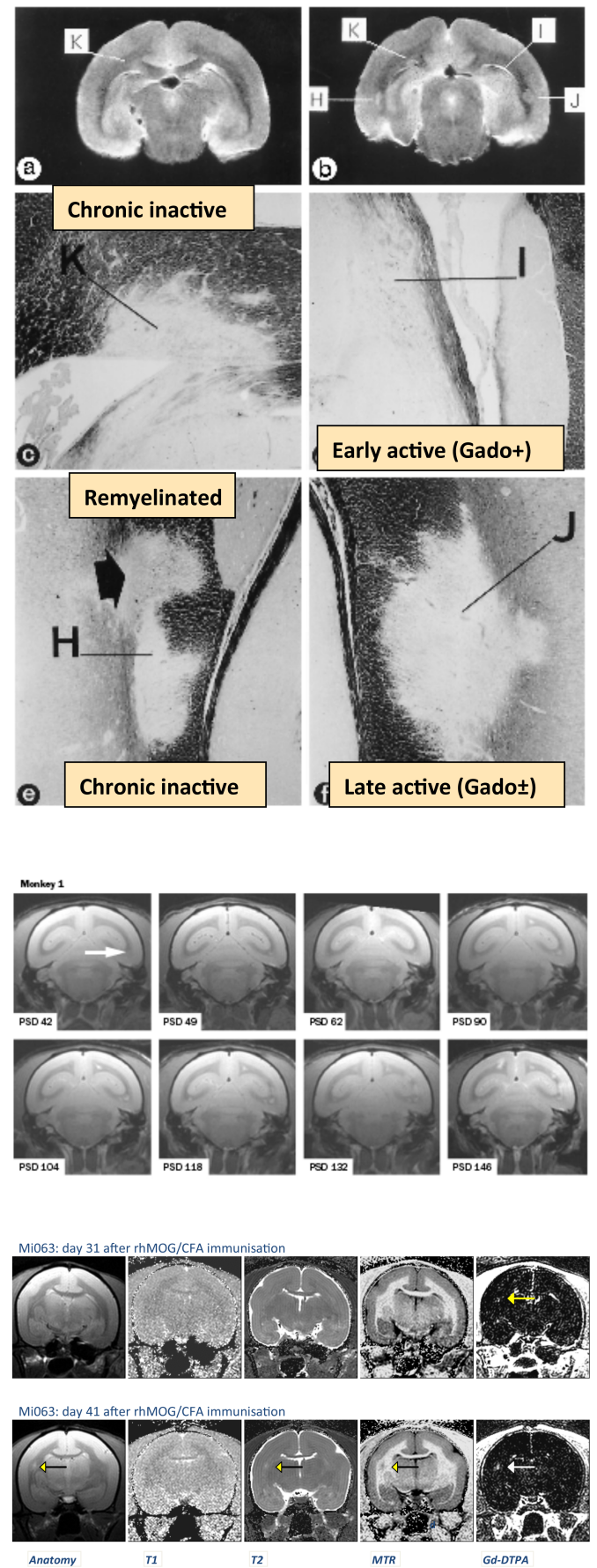

Figure 1. Magnetic resonance imaging of brain lesions in marmoset experimental autoimmune encephalomyelitis (EAE). (a) Marmosets were immunized with human myelin in CFA. The depicted case was sacrificed at the height of the disease with serious clinical deficits. The brain was carefully removed, briefly fixed with $4 \%$ buffered formalin and MRI-scanned (T2-weighted, $4.7 \mathrm{~T}$ ) to detect the spatial distribution of lesions. Lesions are indicated with capitals (a, b). Stainings of individual lesions with Klüver-Barrera for myelin are depicted in (c)-(f). (b) The brain of a single marmoset in which EAE was induced with rhMOG/CFA was longitudinally scanned (T2-weighted, 4.7 T) to illustrate the dissemination of lesion formation in time and space. In the insert the time after immunization (psd: post-sensitization day) is given. (c) Different imaging modalities were developed for in vivo characterization of lesions: T2-weighted images for high contrast anatomy, T1 and T2 relaxation time (RT) images to quantify changes in the signal intensities associated brain injury and inflammation, magnetic transfer ratio (MTR) images which provide essentially the same information and finally images created by subtracting T1-RT images generated before and $10 \mathrm{~min}$ after infusion of the paramagnetic contrast agent gadolinium-DTPA. This modality visualizes lesions with a leaky blood-brain barrier (BBB) caused by inflammation. The message of this figure is that a lesion first visible as a small region of BBB leakage appears 10 days later as a large hyperintense region in the T2 and MTR images. 
a glycosylated protein MOG is tolerogenic, while as a nonglycosylated protein MOG is strongly immunogenic. The latter is illustrated by the recently reported observation that immunization of marmosets with recombinant human MOG (produced in E. coli) formulated with IFA induced full-blown clinical EAE (Jagessar et al., 2015).

We used a panel of synthetic 23-amino-acid-long peptides overlapping by 10 derived from the extracellular domain of human MOG (residues 1-125) to probe the $T$ cell response profile in a genetically heterogeneous population of rhMOGsensitized marmosets (Brok et al., 2000; Kap et al., 2008). The same panel had been used for probing the $T$ cell repertoire of MS patients. The reactivity patterns that we found in EAE-affected marmosets were remarkably similar to the reactivity patterns found by others in MS patients (Kerlero de Rosbo and Ben-Nun, 1998). We observed in monkeys immunized with rhMOG/CFA $T$ cell reactivity against two immuno-dominant $T$ cell epitopes in rhMOG, which are juxtaposed in a highly conserved part of the extracellular domain, residues 24-36 and 40-48. In marmosets immunized with rhMOG/IFA only $T$ cell reactivity against two overlapping peptides that define the first epitope could be detected, namely 14-36 and 24-46 (Jagessar et al., 2015). Neither $T$ cells nor antibodies reacting against the MOG34-56 peptide could be detected. However, marmosets immunized with MOG34-56 peptide in IFA developed full-blown clinical EAE, characterized by MS-like pathology in the white and the grey matter (Jagessar et al., 2010). This observation demonstrates that MOG40-48 specific $T$ cells are present in the normal repertoire. Apparently these highly pathogenic $T$ cells were not activated when the monkeys were immunized with the full-length rhMOG protein in IFA. Where could these effector memory $T$ cell specificities originate from?

In the classical EAE model induced with rhMOG in CFA, CD8 + ve $T$ cells against the MOG40-48 epitope were found to emerge late in the disease, in association with the expression of clinical signs (Kap et al., 2008). This finding indicates pathogenic dominance of this epitope. We further characterized these late-appearing $T$ cells in marmosets immunized with peptide MOG34-56 formulated with IFA, in which clinical signs and widespread demyelination of white and grey matter developed without the induction of myelin-binding autoantibodies (Jagessar et al., 2010). It was shown that the $T$ cells are specific for the MOG40-48 epitope, which is presented via MHC class I/Caja-E molecules; that the responding $T$ cells have an NK-CTL (natural killer-cytotoxic $T$ lymphocyte) phenotype (CD3 + CD8 + CD56+); and that they display cytotoxic activity against EBV-infected $B$ cells pulsed with MOG34-56 (Jagessar et al., 2012a). This type of $T$ cells has been found in humans in the repertoire of effector memory $T$ cells that keep chronic latent infection with CMV under control (Pietra et al., 2003). Interestingly, we also found that the MOG40-48 epitope shares high sequence similarity with a peptide encoded in the UL86 ORF of human
CMV and that $T$ cells raised against the CMV peptide crossreact with MOG34-56 (Brok et al., 2007). As marmosets are naturally infected with a human CMV-related $\beta$-herpesvirus, it is tempting to speculate that the effector memory $T$ cells that cause MS-like disease in marmosets immunized with MOG34-56/IFA originate from the anti-CMV repertoire.

In the EAE model induced with rhMOG in CFA, the sequence 24-36 was defined as the specific epitope of MHC class II/Caja-DRB*W1201 restricted $T$ helper (Th) 1 cells (Brok et al., 2000). The epitope contains at the Asn31 residue the N-linked glycosylation site where the fucosylated glycan epitope of DC-SIGN is attached (Johns and Bernard, 1999). Regarding the putative role of MOG-DC-SIGN interaction in the control of tolerance and autoimmunity (GarciaVallejo et al., 2014; Garcia-Vallejo and van Kooyk, 2009), it is well possible that the anti-MOG24-36 Th cells detected in the rhMOG/IFA model are effector memory regulatory $T$ (Treg) cells present in the normal repertoire. In the presence of danger signals from mycobacteria in CFA these might have been skewed towards a pro-inflammatory Th1 function. Indeed, in marmosets immunized with the long MOG20-50 peptide, which contains both epitopes, formulated with IFA EAE development was suppressed. In these monkeys, $T$ cell and antibody reactivity against the MOG24-36 epitope was clearly detectable, while reactivity against the MOG40-48 epitope was suppressed (Jagessar et al., 2015). By contrast, in monkeys immunized with a mixture of the MOG14-36 and MOG34-56 peptides in IFA, clinical EAE developed and $T$ cell reactivity against both peptides was detectable. This experiment hints at the possibility that Treg cells re-activated by the MOG24-36 epitope may control the activation of CTL against MOG40-48 via linked suppression (Fig. 2).

\section{EAE development in marmosets involves two pathways}

The findings discussed thus far led us to postulate that the core pathogenic process in myelin-immunized marmosets involves two pathways, which develop sequentially but can also be activated separately ('t Hart et al., 2011) (Fig. 3).

The EAE initiation pathway: the observation that marmosets immunized with MOG-deficient myelin develop acute EAE warrants the conclusion that EAE can be initiated independent of autoimmunity against MOG. Indeed, clinical EAE with small-sized lesions of a mainly inflammatory nature that resembled those found in the MBP-induced Lewis rat model could be induced by immunization with MBP or PLP in CFA (Massacesi et al., 1995). In these models Th1 cells are activated, but demyelination is not induced unless anti-MOG antibodies are present (Genain et al., 1995; McFarland et al., 1999). Similarly, EAE initiation in the rhMOG/CFA model initially involves Th1 cell activation and induction of anti-MOG antibodies (Brok et al., 2000). 


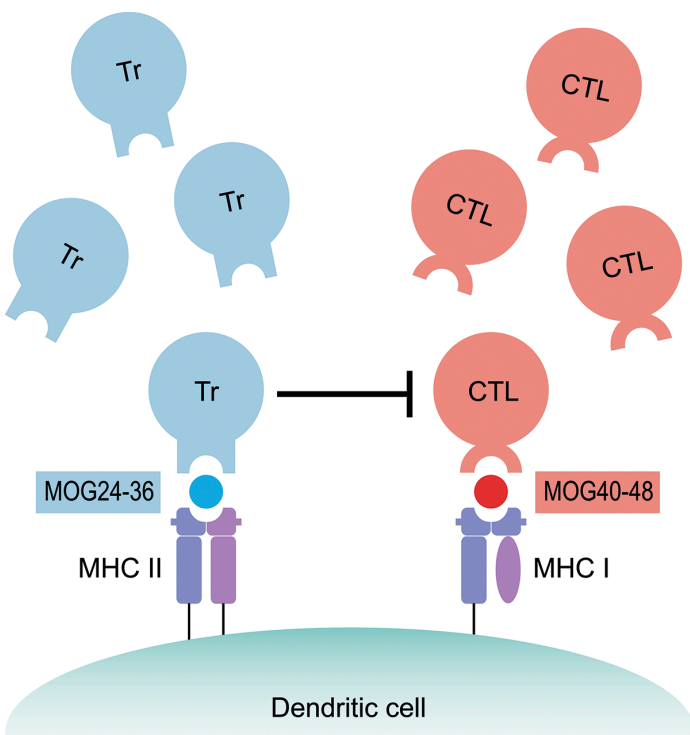

Figure 2. Putative interaction of the immunodominant MOG $T$ cell epitopes. To test the possible interaction of the two MOG $T$ cell epitopes 24-36 and 40-48, we immunized marmoset twins twice (psd 0 and 28) with the long peptide MOG20-50 in which both epitopes were physically linked or with an equimolar mixture of the two short peptides MOG14-36 and MOG34-56 in which the two epitopes were on separate molecules (non-linked). All monkeys were challenged twice (psd 56 and 84) with an encephalitogenic dose of MOG34-56. The adjuvant was in all cases IFA. All twin siblings (3/3) immunized with the non-linked epitopes developed clinically evident EAE, while two of three siblings immunized with the linked epitopes were resistant to EAE. The message of this figure is that $T$ regulator cells ( $\operatorname{Tr} 1)$ specific for MOG24-36 and $T$ effector CTL may need to interact during their reactivation by the same APC.

$B$ cells contribute to the initiation pathway by the production of autoantibodies that bind and opsonize CNS myelin. The immune complexes elicit myelin injury via complemental and/or macrophage-mediated cytotoxicity reactions (ADCC, CDC) (Noseworthy et al., 2000). The observation that sera from marmosets immunized with MOG-deficient myelin do not contain myelin-binding IgG specificities shows that MOG is an important primary target of the antibody opsonization (Jagessar et al., 2008). Antibodies capable of opsonizing myelin bind conformational epitopes (Jagessar et al., 2015; Menge et al., 2007). It has been shown that adoptive transfer of anti-MOG antibodies into MBP/CFAsensitized marmosets induces widespread demyelination of inflammatory lesions (Genain et al., 1995).

The EAE perpetuation pathway: for the progression of EAE, autoimmunity against MOG is essential. NK-CTLs against the MOG epitope 40-48 seem to play an important role in late-stage disease. These $T$ cells can be induced by immunization of marmosets with MOG peptide 34-56 in IFA (Jagessar et al., 2012a), a condition where myelinbinding antibodies are not formed (Jagessar et al., 2015). The pathogenic role of these cytotoxic cells in MS is not exactly clear. It is of considerable interest, however, that a similar type of $T$ cells, albeit specific for another myelin antigen (MBP), has been found in MS lesions, where they were found engaged in the killing of HLA-E+ ve oligodendrocytes (Zaguia et al., 2013).

In the EAE perpetuation pathway $B$ cells have en essential pathogenic role, but not via the production of autoantibodies. Instead $B$ cells have an essential role in the activation of the autoaggressive CTLs that cause direct demyelination ('t Hart et al., 2013).

\section{Validation of the marmoset EAE model with clinically relevant immunotherapies}

The evolutionary distance between marmosets and humans has been estimated at \pm 55 million years. This relative proximity in comparison with mice is reflected by the relatively high cross-reaction of mAbs raised against $\mathrm{CD}$ markers of human leukocytes with leukocytes of marmosets (Jagessar et al., 2013a; Brok et al., 2001). This makes the marmoset a potentially useful model for the safety and efficacy evaluation of biological therapeutics, mAbs for example, which for specificity reasons cannot be tested in lower species. The term "potentially useful" is used here on purpose as crossreaction of a test compound alone is not a sufficient criterion for a translationally relevant preclinical model of MS. The model will only be relevant for MS when the immunopathogenic mechanisms resemble those in the human disease. One way to find this out is to test the activity of treatments, which have proven effective or not effective in mouse EAE or RRMS, also in the marmoset model. Figure 4 gives a graphical representation of this strategy.

\subsection{Treatments targeting $T$ cells}

Rodent EAE models show a central pathogenic role of CD4 $+T$ cells. This concept prompted a small-sized clinical trial in which the anti-human CD4 mAb MT-412 was tested in RRMS patients (van Oosten et al., 1997). The treatment induced profound depletion of CD $4+T$ cells but nevertheless exerted no clear effect on lesion activity detected with MRI, suggesting that CD4 $+T$ cells may have a different pathogenic role in RRMS than in the EAE model.

The differentiation of CD4 $+T$ cells into functionally polarized pro-inflammatory subtypes (Th1 and Th17) is steered by two related cytokines, IL-12 and IL-23. Both cytokines are heterodimers, composed of a shared p40 subunit and a specific subunit, respectively p35 and p19. Ustekinumab is a fully human $\mathrm{mAb}$ directed against the shared $\mathrm{p} 40$ subunit of human IL-12/IL-23. The mAb binds the cytokines from marmosets but not from lower species, precluding ef- 


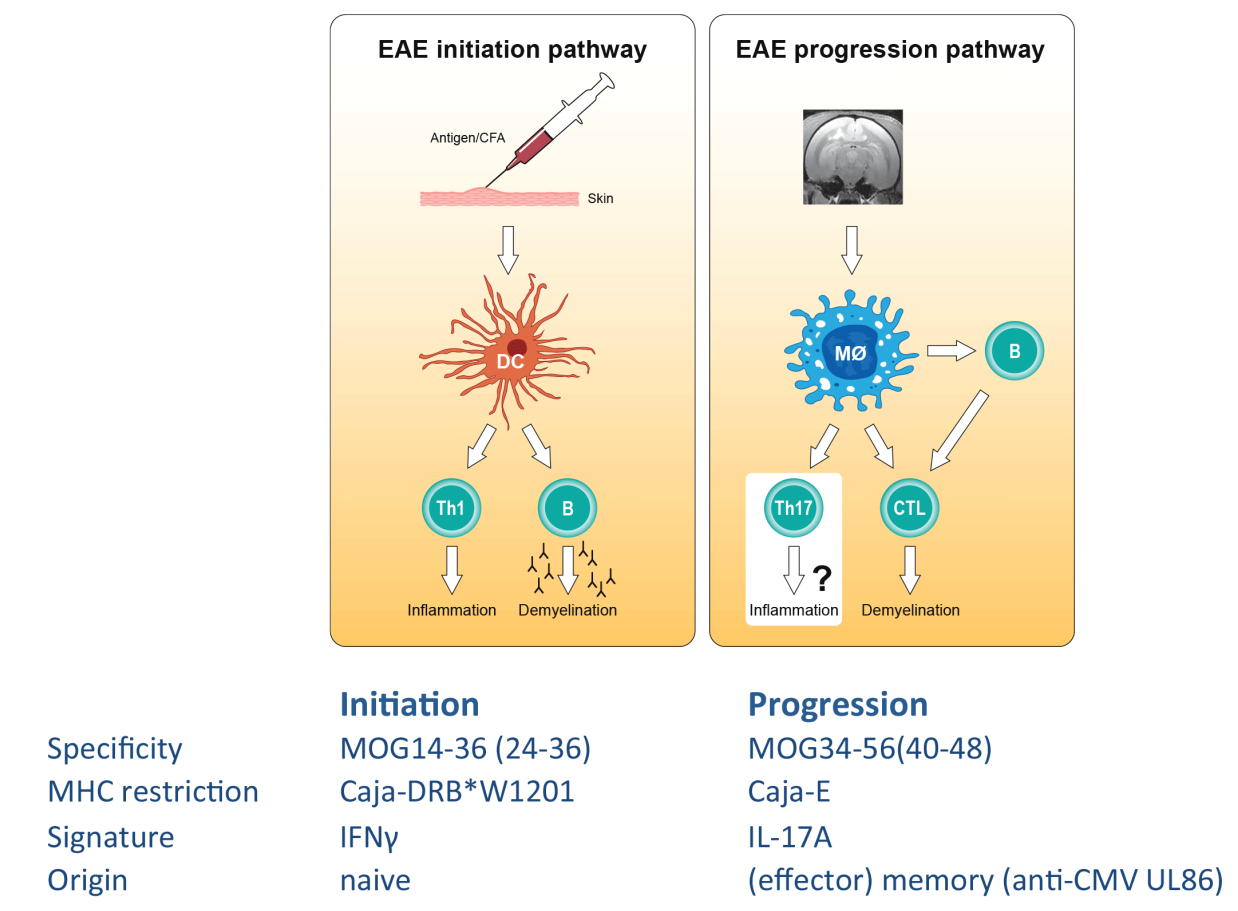

Figure 3. The two pathogenic pathways in the rhMOG/CFA-induced marmoset experimental autoimmune encephalomyelitis (EAE) model. In all marmosets immunized with rhMOG/CFA, initiation of EAE involves the activation of CD4 $T$ helper 1 cells specific for the epitope 24-36. Activation of the $T$ cells is restricted by the monomorphic allele Caja-DRB*W1201, which all marmosets have in common. After a variable period of time CD4 + CD8 + CD56 + cytotoxic $T$ cells specific for the epitope 40-48 are activated; activation is restricted by the invariant non-classical MHC class Ib allele Caja-E, which all marmosets have in common.

ficacy testing in rodent EAE models. Unexpectedly, the mAb lacked clinical efficacy in RRMS (Segal et al., 2008), but it has found a new clinical target in psoriasis and possibly inflammatory bowel disease. In a first experiment marmosets immunized with human myelin in CFA were treated with the $\mathrm{mAb}$ from 1 day before the immunization, in contrast to RRMS, resulting in complete protection against EAE (Brok et al., 2002). This encouraging finding prompted us to test the $\mathrm{mAb}$ also in a clinically more relevant study design, namely starting during the chronic phase of the disease ('t Hart et al., 2005). In brief, marmosets immunized with rhMOG/CFA were subjected to T2-weighted brain MRI to visualize whitematter lesions, as depicted in Fig. 1b. Once lesions of sufficient size for quantification of inflammatory activity were detected, treatment with the antibody or placebo was started. We observed that - although the increase of T2 signal intensity, as a measure of inflammation, and of the lesion enlargement, as a measure of demyelination, were completely suppressed - the onset of clinical signs was only temporarily delayed, similar to RRMS. It is tempting to speculate that the delayed exacerbation of clinical signs, while MRI-detectable lesion inflammation appears suppressed, is due to demyelination induced by the autoaggressive CTLs, which drive the EAE perpetuation pathway (see above). Importantly, these studies highlight the profound need for EAE study design to be as clinically relevant as possible and further provide ev- idence that the marmoset EAE model is a valid pre-clinical model with the correct study design.

The signature and immuno-active cytokine of Th1 cells secreted after stimulation with IL-12 is interferon (IFN) $\gamma$. Clinical studies in RRMS showed that administration of IFN $\gamma$ exacerbated disease activity (Panitch et al., 1987) while treatment with anti-IFN $\gamma$ antibody had only minor clinical effects. In the mouse EAE model both positive and negative effects of IFN $\gamma$ neutralization have been reported, often depending on the timing of the treatment (Sanvito et al., 2010). We have tested whether early (psd 025 ) or late (psd 56-81) treatment with IFN $\gamma$ would alter the EAE course in marmosets immunized with huMOG3456 peptide in IFA. We observed that neither of the treatment regimens had a positive effect on the disease course, but the disease exacerbation observed in RRMS was not seen. Remarkably,Th1-associated humoral and cellular autoimmune parameters were affected (Jagessar et al., 2012b).

The signature cytokine of Th17 cells is IL-17A, but the pathogenic role of the cytokine in EAE is unclear. In the rhMOG/CFA marmoset EAE model, IL-17A is prominently expressed by the late-acting CTLs, which induce cortical grey-matter demyelination and irreversible neurological deficit (Jagessar et al., 2010). We tested a $\operatorname{IgG} 4 \kappa \mathrm{mAb}$ raised against human IL-17A in the rhMOG/CFA marmoset EAE model (Kap et al., 2011a). Treatment with the mAb was 


\section{Validation of preclinical models}

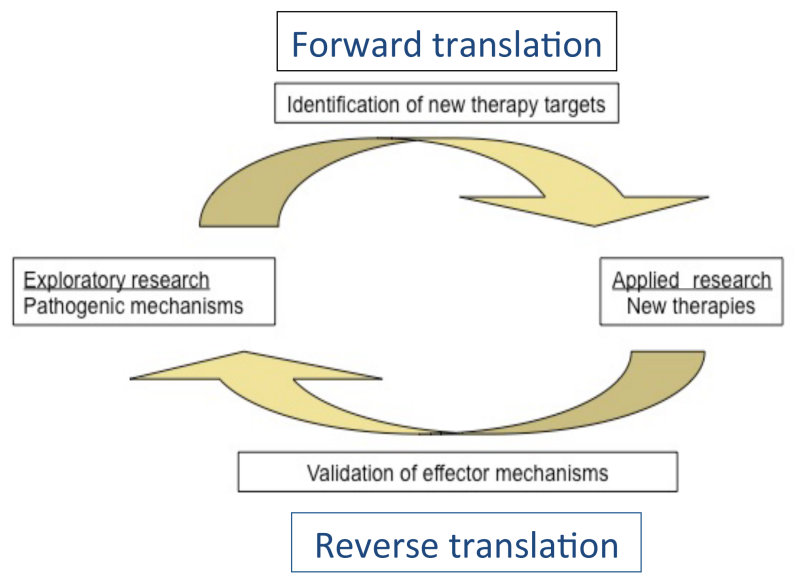

Figure 4. Validation of the marmoset experimental autoimmune encephalomyelitis (EAE) model. Therapies showing promising clinical effects in the EAE model, of mouse and marmosets alike, are tested in MS patients. When this forward translation proves successful, it confirms the validity of the targeted pathogenic process EAE model for MS. In the case of forward translation showing that promising clinical effects observed in MS cannot be reproduced in MS, the treatment should be re-evaluated in the EAE model to examine why the targeted process seems irrelevant for MS.

started 1 day before the immunization and continued to the end of the experiment. The antibody caused only a moderate delay of the disease onset.

IL-7 is a hemopoietic growth factor that is produced by stromal cells in bone marrow and thymus and has a crucial role in lymphopoiesis. In the periphery IL-7 is produced by a variety of cells, including APCs (dendritic cells). IL7 signals through CD127 for maintenance of $T$ and $B$ cell homeostasis. Blockade of IL-7 binding to CD127 ameliorates disease in mouse EAE models and is currently under evaluation in RRMS patients (personal communication). Importantly, EBV-infected $B$ cells, which are the requisite APCs for the late-acting autoaggressive CTLs in the rhMOG/CFA marmoset EAE model, produce high levels of IL-7. These findings prompted us to test a new chimeric $\mathrm{IgG} 1 \kappa \mathrm{mAb}$ against human CD127 in marmosets immunized with MOG34-56/IFA (Dunham et al., 2016). Unexpectedly, we observed a dichotomous clinical effect of the treatment. In twins with an early disease onset, we observed a markedly prolonged asymptomatic period in the mAbtreated sibling, whereas in twins with a late disease onset no evident clinical effect was found. It is unclear how IL7R blockade will perform in the clinic, although it has been suggested that IL-7R may be an attractive target for a subset of MS patients. Interestingly, we found in monkeys responding to the treatment that the increase of circulating $\mathrm{CD} 20+\mathrm{CD} 40+B$ cells induced by the immunization was suppressed. This subpopulation was also prominently de- pleted by the anti-CD20 mAb but spared in the monkeys treated with anti-BlyS/APRIL (see below). This led us to postulate that among the $\mathrm{CD} 20+\mathrm{veCD} 40+$ ve $B$ cells there are the virus-infected APCs of the autoaggressive CTLs.

Collectively, these observations corroborate the concept that the pathogenic role of pro-inflammatory CD4 + Th1/Th17 cells is confined to EAE initiation, whereas disease progression is driven by a different pathogenic process. Nevertheless, several tested treatments failed to reproduce promising effects observed in mouse EAE when they were tested in the marmoset EAE model and validate the marmoset EAE model as a pre-clinical model for MS. One explanation for these paradoxical effects may be that the immune process most closely linked with the induction of neurological problems is the activation of autoaggressive effector memory CTLs. These cells may already have been committed to their pathogenic function and may be refractory to immunomodulatory treatment. For this reason, we became interested in the $B$ cells that are responsible for the activation of the CTLs.

\subsection{Treatments targeting $B$ cells}

CD20 is a broadly expressed surface molecule in the $B$ cell lineage. Only pro- $B$ cells and terminally differentiated plasma cells, which are the main producers of antibodies, do not express CD20. The collective results of clinical trials with three mAbs against human CD20, one chimeric (rituximab) and two fully human (ofatumumab and ocrelizumab), show a remarkably brisk clinical effect, which seems to persist for months (Barun and Bar-Or, 2012). However, as plasma levels of antibodies capable to mediate demyelination were not affected, the exact working mechanism is not understood.

HuMab7D8 is a clonal variant of ofatumumab that is also fully human $(\operatorname{IgG} 1 \kappa)$ and cross-reacts well with marmoset $B$ cells. The mAb was tested in the rhMOG/CFA marmoset EAE model starting well after the single immunization (psd 21) (Kap et al., 2010). The treatment induced a similar brisk and persistent depletion of $B$ cells to that observed in RRMS patients. Moreover, robust and persistent suppression of clinical signs was observed. Detailed examination of the effect on EAE pathology showed that $B$ cells were also eliminated from the brain, while the formation of MS-like lesions in the white and grey matter of brain and spinal cord was markedly reduced (Kap et al., 2011b). A clear difference with the RRMS clinical trial was that in the EAE model plasma levels of autoantibodies binding MOG protein, potentially capable of mediating demyelination, were suppressed. This prompted us to perform an additional experiment in the marmoset EAE model induced with MOG34-56/IFA in which MOG protein-binding antibodies are not formed. Also in this model we observed a robust and persistent beneficial effect of anti-CD20 mAb, both on the clinical signs and on the underlying pathology (Jagessar et al., 2012c). The inevitable conclusion of this experiment is that $B$ cells are directly in- 
volved in the activation and/or function of the autoaggressive NK-CTLs that drive EAE development in this model.

The survival and differentiation of $B$ cells depend on the cytokines BAFF/BlyS and APRIL (Jagessar et al., 2012). The two cytokines are physiologically active as BAFF-BAFF or APRIL-APRIL homodimers or as BAFF-APRIL heterodimer. There are three receptors for these cytokines expressed on $B$ cells: BAFF-R, BCMA, and TACI. BAFF-R is only bound by BAFF, while both homodimers bind to BCMA and TACI, but only TACI binds the heterodimer. Regarding the impressive clinical effect in RRMS of $B$ cell depletion via anti-CD20 mAb, it was a reasonable expectation that $B$ cell depletion via the capture of BlyS/BAFF and/or APRIL might also have a beneficial clinical effect. However, this was not observed as a clinical trial in RRMS with atacicept; a recombinant fusion protein of human TACI and IgG unexpectedly increased MRI-detectable lesion activity despite the depletion of peripheral $B$ cells (Kappos et al., 2014).

We used a somewhat different approach in the marmoset EAE model to replicate these findings, namely using a mAb against human BAFF (belimumab) and a mAb against human APRIL (Jagessar et al., 2012d). We observed that both mAbs caused only a moderate, albeit statistically significant, delay of the disease onset in marmosets immunized with rhMOG/CFA.

The reason for the unexpected paradoxical clinical effects of $B$ cell depletion via anti-CD20 or inactivation of BlyS or APRIL is not known but seems not restricted to MS. Also in rheumatoid arthritis the anti-CD20 mAb rituximab exerted a remarkable clinical effect, whereas atacicept and the anti-BlyS mAb belimumab were only marginally effective (Dorner et al, 2010; Richez et al., 2014). A possible explanation was found in the marmoset EAE model. We observed that in monkeys treated with anti-CD20 mAb the copy number of CalHV3 DNA in lymph nodes and spleen was strongly reduced while the copy number was increased in monkeys treated with anti-BlyS or anti-APRIL mAb (Jagessar et al., 2013b). Our interpretation of this finding is that $B$ cells immortalized by the EBV-related $\gamma$-herpesvirus CalHV3 may not need BAFF or APRIL for their survival and thus ignore inactivation of the cytokines, while they express CD20 and can thus still be depleted with anti-CD20 mAb.

Further research in the model also provided a possible explanation for the brisk clinical effect of anti-CD20 mAb. We observed that the space left inside lymph nodes by the depletion of CD20+ ve $B$ cells is replenished by activated $T$ cells expressing the lymph node homing receptor CCR7. This was not observed in monkeys treated with anti-BlyS or anti-APRIL mAb (Kap et al., 2014). This finding may suggest that the anti-CD20 treatment may interfere with the licensing of activated autoreactive $T$ cells to egress the lymph nodes.

A marker that is constitutively expressed on $B$ cells and induced after activation on myeloid APCs is CD40 (Laman et al., 1996; van Kooten and Banchereau, 1997). As mentioned earlier, the CD20 + APC of the core pathogenic autoaggressive CTLs prominently express CD40. The ligation of CD40 on $B$ cells with its counter structure on $T$ cells (CD154) elicits bi-directional signaling that is important for $B$ cell activation and differentiation; antibody isotype switch also depends on CD40-CD154 interaction. In EBV-infected $B$ cells the major oncoprotein LMP1 mimics constitutively activated CD40, but recent data suggest that activation signals can still be relayed through CD40 (Ma et al., 2015). In agreement, we observed that incubation of EBV-infected marmoset $B$ cells with a stimulatory anti-CD40 mAb enhances IgG production (own unpublished observation).

The $\mathrm{mAb} 5 \mathrm{D} 12$ is a non-stimulatory mouse IgG2b against human CD40 that binds marmoset CD40 and blocks its interaction with human CD40 ligand/CD154 (Laman et al., 2002). Marmosets immunized with human myelin/CFA were treated early (psd 14-42) or later (psd 25-53) with the mAb. The treatment suppressed the expression of EAE symptoms during presence of the mAb but did not abrogate EAE development after the treatment was stopped, which might be due to the development of a neutralizing antibody response (Laman et al., 2002). For this reason, a second experiment was performed testing a novel mouse-human chimeric version of 5D12 in the rhMOG/CFA model. Also in the mAbtreated monkeys clinical signs did not develop during the 40day observation period, while all placebo-treated monkeys displayed clinical EAE (Boon et al., 2001). Importantly, the treatment effect was reflected by alteration of the antibody reactivity profile with a panel of rhMOG peptides.

\section{Concluding remarks}

The marmoset EAE model tells a different story about the putative immunopathogenesis of human multiple sclerosis than the corresponding models in inbred/SPF mice. The most important lesson is that the pathogen-educated $T$ cell repertoire of marmosets contains effector memory cytotoxic specificities that can readily be activated by an antigen without the need of danger co-signaling. These CTLs mediate a pathogenic pathway that has not (yet) been found in mouse EAE models but that seem to be relevant for ongoing disease in MS patients. The finding that the EBV-infected $B$ cells are involved in the activation of these highly pathogenic cells may provide a mechanistic explanation for the elusive association of this $\gamma 1$ herpesvirus with susceptibility to MS (Lunemann and Munz, 2009; Pakpoor et al., 2012). Another important finding has been that in the marmoset EAE model MS-like cortical grey-matter pathology develops. Essentially all lesion stages present in the grey matter of MS patients can be found in marmosets (Pomeroy et al., 2005). Moreover, at a late disease stage also grey-matter atrophy develops (Pomeroy et al., 2008). This implies that the marmoset provides a highly desired experimental model for the study of 
grey-matter pathology development in progressive MS (Mahad et al., 2015).

Acknowledgements. The authors would like to thank Henk van Westbroek for the artwork.

Edited by: E. Fuchs

Reviewed by: two anonymous referees

\section{References}

Arnon, R., Sela, M., and Teitelbaum, D.: New insights into the mechanism of action of copolymer 1 in experimental allergic encephalomyelitis and multiple sclerosis, J. Neurol., 243, 8-13, 1996.

Ascherio, A. and Munger, K. L.: Environmental risk factors for multiple sclerosis. Part I: the role of infection, Ann. Neurol., 61, 288299, 2007a.

Ascherio, A. and Munger, K. L.: Environmental risk factors for multiple sclerosis. Part II: Noninfectious factors, Ann. Neurol., 61, 504-513, 2007b.

Ascherio, A. and Munger, K. L.: EBV and Autoimmunity, Curr. Top. Microbiol. Immunol., 390, 365-385, 2015.

Ascherio, A., Munger, K. L., and Lunemann, J. D.: The initiation and prevention of multiple sclerosis, Nat. Rev. Neurol., 8, 602612, 2012.

Barkhof, F., Simon, J. H., Fazekas, F., Rovaris, M., Kappos, L., de Stefano, N., Polman, C. H., Petkau, J., Radue, E. W., Sormani, M. P., Li, D. K., O'Connor, P., Montalban, X., Miller, D. H., and Filippi, M.: MRI monitoring of immunomodulation in relapseonset multiple sclerosis trials, Nat. Rev. Neurol., 8, 13-21, 2012.

Barun, B. and Bar-Or, A.: Treatment of multiple sclerosis with AntiCD20 antibodies, Clin. Immunol., 142, 31-37, 2012.

Billiau, A. and Matthys, P.: Modes of action of Freund's adjuvants in experimental models of autoimmune diseases, J. Leukoc. Biol., 70, 849-860, 2001.

Boon, L., Brok, H. P., Bauer, J., Ortiz-Buijsse, A., Schellekens, M. M., Ramdien-Murli, S., Blezer, E., van Meurs, M., Ceuppens, J., de Boer, M., 't Hart, B. A., and Lamanm, J. D.: Prevention of experimental autoimmune encephalomyelitis in the common marmoset (Callithrix jacchus) using a chimeric antagonist monoclonal antibody against human CD40 is associated with altered $B$ cell responses, J. Immunol., 167, 2942-2949, 2001.

Brok, H. P., Uccelli, A., Kerlero De Rosbo, N., Bontrop, R. E., Roccatagliata, L., de Groot, N. G., Capello, E., Laman, J. D., Nicolay, K., Mancardi, G. L., Ben-Nun, A., and 't Hart, B. A.: Myelin/oligodendrocyte glycoprotein-induced autoimmune encephalomyelitis in common marmosets: the encephalitogenic $T$ cell epitope pMOG24-36 is presented by a monomorphic MHC class II molecule, J. Immunol., 165, 1093-1101, 2000.

Brok, H. P., Hornby, R. J., Griffiths, G. D., Scott, L. A., and 't Hart, B. A.: An extensive monoclonal antibody panel for the phenotyping of leukocyte subsets in the common marmoset and the cotton-top tamarin, Cytometry, 45, 294-303, 2001.

Brok, H. P., Van Meurs, M., Blezer, E., Schantz, A., Peritt, D., Treacy, G., Laman, J. D., Bauer, J., and 't Hart, B.: Prevention of experimental autoimmune encephalomyelitis in common marmosets using an anti-IL-12p40 monoclonal antibody, J. Immunol., 169, 6554-6563, 2002.

Brok, H. P., Boven, L., van Meurs, M., Kerlero de Rosbo, N., Celebi-Paul, L., Kap, Y. S., Jagessar, A., Hintzen, R. Q., Keir, G., Bajramovic, J., Ben-Nun, A., Bauer, J., Laman, J. D., Amor, S., and 't Hart, B. A.: The human CMV-UL86 peptide 981-1003 shares a crossreactive $T$-cell epitope with the encephalitogenic MOG peptide $34-56$, but lacks the capacity to induce EAE in rhesus monkeys, J. Neuroimmunol., 182, 135-152, 2007.

Cho, Y., Ramer, J., Rivailler, P., Quink, C., Garber, R. L., Beier, D. R., and Wang, F.: An Epstein-Barr-related herpesvirus from marmoset lymphomas, P.Natl. Acad. Sci. USA, 98, 1224-1229, 2001.

Collins, B. E., Yang, L. J., Mukhopadhyay, G., Filbin, M. T., Kiso, M., Hasegawa, A., and Schnaar, R. L.: Sialic acid specificity of myelin-associated glycoprotein binding, J. Biol. Chem., 272, 1248-1255, 1997.

Davies, L. R. and Varki, A.: Why Is N-Glycolylneuraminic Acid Rare in the Vertebrate Brain?, Top Curr Chem., 366, 31-54, doi:10.1007/128_2013_419,2013.

Davis, M. M.: A prescription for human immunology, Immunity, 29, 835-838, 2008.

Dendrou, C. A., Fugger, L., and Friese, M. A.: Immunopathology of multiple sclerosis, Nat. Rev. Immunol., 15, 545-558, 2015.

de Vos, A. F., van Meurs, M., Brok, H. P., Boven, L. A., Hintzen, R. Q., van der Valk, P., Ravid, R., Rensing, S., Boon, L., 't Hart, B. A., and Laman, J. D.: Transfer of central nervous system autoantigens and presentation in secondary lymphoid organs, J. Immunol., 169, 5415-5423, 2002.

Dorner, T., Kinnman, N., and Tak, P. P.: Targeting $B$ cells in immune-mediated inflammatory disease: a comprehensive review of mechanisms of action and identification of biomarkers, Pharmacol. Therapeut., 125, 464-475, 2010.

Dunham, J., Lee, L. F., van Driel, N., Laman, J. D., Ni, I., Zhai, W., Tu, G. H., Lin, J. C., Bauer, J., t Hart, B. A., and Kap, Y. S.: Blockade of CD127 Exerts a Dichotomous Clinical Effect in Marmoset Experimental Autoimmune Encephalomyelitis, J. Neuroim. Pharmacol., 1, 73-83, doi:10.1007/s11481-015-96296, 2016.

Fabriek, B. O., Zwemmer, J. N., Teunissen, C. E., Dijkstra, C. D., Polman, C. H., Laman, J. D., and Castelijns, J. A.L In vivo detection of myelin proteins in cervical lymph nodes of MS patients using ultrasound-guided fine-needle aspiration cytology, J. Neuroimmunol., 161, 190-194, 2005.

Garcia-Vallejo, J. J. and van Kooyk, Y.: Endogenous ligands for Ctype lectin receptors: the true regulators of immune homeostasis, Immunol. Rev., 230, 22-37, 2009.

Garcia-Vallejo, J. J., Ilarregui, J. M., Kalay, H., Chamorro, S., Koning, N., Unger, W. W., Ambrosini, M., Montserrat, V., Fernandes, R. J., Bruijns, S. C., van Weering, J. R., Paauw, N. J., O'Toole, T., van Horssen, J., van der Valk, P., Nazmi, K., Bolscher, J. G., Bajramovic, J., Dijkstra, C. D., 't Hart, B. A., and van Kooyk, Y.: CNS myelin induces regulatory functions of DC-SIGN-expressing, antigen-presenting cells via cognate interaction with MOG, J. Exp. Med., 211, 1465-1483, 2014.

Genain, C. P., Nguyen, M. H., Letvin, N. L., Pearl, R., Davis, R. L., Adelman, M., Lees, M. B., Linington, C., and Hauser, S. L.: Antibody facilitation of multiple sclerosis-like lesions in a nonhuman primate, J. Clin. Invest., 96, 2966-2974, 1995. 
Haig, D.: What is a marmoset?, Am. J. Primatol., 49, 285-296, 1999.

Jagessar, S. A., Smith, P. A., Blezer, E., Delarasse, C., Pham-Dinh, D., Laman, J. D., Bauer, J., Amor, S., and 't Hart, B.: Autoimmunity against myelin oligodendrocyte glycoprotein is dispensable for the initiation although essential for the progression of chronic encephalomyelitis in common marmosets, J. Neuropathol. Exp. Neurol., 67, 326-340, 2008.

Jagessar, S. A., Kap, Y. S., Heijmans, N., van Driel, N., van Straalen, L., Bajramovic, J. J., Brok, H. P., Blezer, E. L., Bauer, J., Laman, J. D., and 't Hart, B. A.: Induction of progressive demyelinating autoimmune encephalomyelitis in common marmoset monkeys using MOG34-56 peptide in incomplete freund adjuvant, J. Neuropathol. Exp. Neurol., 69, 372-385, 2010.

Jagessar, S. A., Heijmans, N., Blezer, E. L., Bauer, J., Blokhuis, J. H., Wubben, J. A., Drijfhout, J. W., van den Elsen, P. J., Laman, J. D., and 't Hart, B. A.: Unravelling the $T$-cell-mediated autoimmune attack on CNS myelin in a new primate EAE model induced with MOG34-56 peptide in incomplete adjuvant, Eur. J. Immunol., 42, 217-227, 2012a.

Jagessar, S. A., Gran, B., Heijmans, N., Bauer, J., Laman, J. D., 't Hart, B. A., and Constantinescu, C. S.: Discrepant effects of human interferon-gamma on clinical and immunological disease parameters in a novel marmoset model for multiple sclerosis, $\mathrm{J}$. Neuroim. Pharmacol., 7, 253-265, 2012 b.

Jagessar, S. A., Heijmans, N., Bauer, J., Blezer, E. L., Laman, J. D., Hellings, N., and 't Hart, B. A.: B-cell depletion abrogates $T$ cell-mediated demyelination in an antibody-nondependent common marmoset experimental autoimmune encephalomyelitis model, J. Neuropathol. Exp. Neurol., 71, 716-728, 2012c.

Jagessar, S. A., Heijmans, N., Oh, L., Bauer, J., Blezer, E. L., Laman, J. D., Migone, T. S., Devalaraja, M. N., and 't Hart, B. A.: Antibodies against human BLyS and APRIL attenuate EAE development in marmoset monkeys, J. Neuroim. Pharmacol., 7, 557-570, 2012d.

Jagessar, S. A., Vierboom, M., Blezer, E. L., Bauer, J., 't Hart, B. A., and Kap, Y. S.: Overview of models, methods, and reagents developed for translational autoimmunity research in the common marmoset (Callithrix jacchus), Experimental animals/Japanese Association for Laboratory Animal Science, 62, 159-171, 2013 a.

Jagessar, S. A., Fagrouch, Z., Heijmans, N., Bauer, J., Laman, J. D., Oh, L., Migone, T., Verschoor, E. J., and 't Hart, B. A.: The different clinical effects of anti-BLyS, anti-APRIL and anti-CD20 antibodies point at a critical pathogenic role of gamma-herpesvirus infected $B$ cells in the marmoset EAE model, J. Neuroim. Pharmacol., 8, 727-738, 2013b.

Jagessar, S. A., Heijmans, N., Blezer, E. L., Bauer, J., Weissert, R., and $t$ Hart, B. A.: Immune profile of an atypical EAE model in marmoset monkeys immunized with recombinant human myelin oligodendrocyte glycoprotein in incomplete Freund's adjuvant, J. Neuroinflam., 12, 169-186, 2015.

Johns, T. G. and Bernard, C. C.: The structure and function of myelin oligodendrocyte glycoprotein, J. Neurochem., 72, 1-9, 1999.

Kap, Y. S., Smith, P., Jagessar, S. A., Remarque, E., Blezer, E., Strijkers, G. J., Laman, J. D., Hintzen, R. Q., Bauer, J., Brok, H. P., and t Hart, B. A.: Fast progression of recombinant human myelin/oligodendrocyte glycoprotein (MOG)-induced experimental autoimmune encephalomyelitis in marmosets is as- sociated with the activation of MOG34-56-specific cytotoxic $T$ cells, J. Immunol., 180, 1326-1337, 2008.

Kap, Y. S., van Driel, N., Blezer, E., Parren, P. W., Bleeker, W. K., Laman, J. D., Craigen, J. L., and 't Hart, B. A.: Late $B$ cell depletion with a human anti-human CD20 IgG1kappa monoclonal antibody halts the development of experimental autoimmune encephalomyelitis in marmosets, J. Immunol., 185, 3990-4003, 2010.

Kap, Y. S., Jagessar, S. A., van Driel, N., Blezer, E., Bauer, J., van Meurs, M., Smith, P., Laman, J. D., and t Hart, B. A.: Effects of early IL-17A neutralization on disease induction in a primate model of experimental autoimmune encephalomyelitis, J. Neuroim. Pharmacol., 6, 341-353, 2011 a.

Kap, Y. S., Bauer, J., Driel, N. V., Bleeker, W. K., Parren, P. W., Kooi, E. J., Geurts, J. J., Laman, J. D., Craigen, J. L., Blezer, E., and 't Hart, B. A.: B-Cell Depletion Attenuates White and Gray Matter Pathology in Marmoset Experimental Autoimmune Encephalomyelitis, J. Neuropathol. Exp. Neurol., 70, 992-1005, $2011 b$.

Kap, Y. S., van Driel, N., Laman, J. D., Tak, P. P., and t Hart, B. A.: CD20 $+B$ Cell Depletion Alters $T$ Cell Homing, J. Immunol., 192, 4242-4253, 2014.

Kappos, L., Hartung, H. P., Freedman, M. S., Boyko, A., Radu, E. W., Mikol, D. D., Lamarine, M., Hyvert, Y., Freudensprung, U., Plitz, T., van Beek, J., and Group, A. S.: Atacicept in multiple sclerosis (ATAMS): a randomised, placebo-controlled, doubleblind, phase 2 trial, Lancet Neurol., 13, 353-363, 2014.

Kerlero de Rosbo, N. K. and Ben-Nun, A.: T-cell responses to myelin antigens in multiple sclerosis; relevance of the predominant autoimmune reactivity to myelin oligodendrocyte glycoprotein, J. Autoimmun., 11, 287-299, 1998.

Laman, J. D. and Weller, R. O.: Drainage of cells and soluble antigen from the CNS to regional lymph nodes, J. Neuroim. Pharmacol., 8, 840-856, 2013.

Laman, J. D., Claassen, E., and Noelle, V: Functions of CD40 and its ligand, gp39 (CD40L), Crit. Rev. Immunol., 16, 59-108, 1996.

Laman, J. D., 't Hart, B. A., Brok, H., Meurs, M., Schellekens, M. M., Kasran, A., Boon, L., Bauer, J., Boer, M., and Ceuppens, J.: Protection of marmoset monkeys against EAE by treatment with a murine antibody blocking CD40 (mu5D12), Eur. J. Immunol., 32, 2218-2228, 2002.

Lunemann, J. D. and Munz, C. EBV in MS: guilty by association?, Trends Immunol., 30, 243-248, 2009.

Ma, S. D., Xu, X., Plowshay, J., Ranheim, E. A., Burlingham, W. J., Jensen, J. L., Asimakopoulos, F., Tang, W., Gulley, M. L., Cesarman, E., Gumperz, J. E., and Kenney, S. C.: LMP1-deficient Epstein-Barr virus mutant requires $\mathrm{T}$ cells for lymphomagenesis, J. Clin. Invest., 125, 304-315, 2015.

Mahad, D. H., Trapp, B. D., and Lassmann, H.: Pathological mechanisms in progressive multiple sclerosis, Lancet Neurol., 14, 183193, 2015.

Massacesi, L., Genain, C. P., Lee-Parritz, D., Letvin, N. L., Canfield, D., and Hauser, S. L.: Active and passively induced experimental autoimmune encephalomyelitis in common marmosets: a new model for multiple sclerosis, Ann. Neurol., 37, 519-530, 1995.

McFarland, H. I., Lobito, A. A., Johnson, M. M., Nyswaner, J. T., Frank, J. A., Palardy, G. R., Tresser, N., Genain, C. P., Mueller, 
J. P., Matis, L. A., and Lenardo, M. J.: Determinant spreading associated with demyelination in a nonhuman primate model of multiple sclerosis, J. Immunol., 162, 2384-2390, 1999.

Menge, T., von Budingen, H. C., Lalive, P. H., and Genain, C. P.: Relevant antibody subsets against MOG recognize conformational epitopes exclusively exposed in solid-phase ELISA, Eur. J. Immunol., 37, 3229-3239, 2007.

Nigida, S. M., Falk, L. A., Wolfe, L. G., and Deinhardt, F.: Isolation of a cytomegalovirus from salivary glands of white-lipped marmosets (Saguinus fuscicollis), Lab. Anim. Sci., 29, 53-60, 1979.

Noseworthy, J. H., Lucchinetti, C., Rodriguez, M., and Weinshenker, B. G.: Multiple sclerosis, N. Engl. J. Med., 343, 938952, 2000

Pakpoor, J., Disanto, G., Gerber, J. E., Dobson, R., Meier, U. C., Giovannoni, G., and Ramagopalan, S. V.: The risk of developing multiple sclerosis in individuals seronegative for Epstein-Barr virus: a meta-analysis, Mult Scler., 19, 162-166, doi:10.1177/1352458512449682, 2012.

Pakpoor, J., Disanto, G., Gerber, J. E., Dobson, R., Meier, U. C., Giovannoni, G., and Ramagopalan, S. V.: The risk of developing multiple sclerosis in individuals seronegative for Epstein-Barr virus: a meta-analysis, Mult. Scler., 19, 162-166, 2013.

Panitch, H. S., Hirsch, R. L., Haley, A. S., and Johnson, K. P.: Exacerbations of multiple sclerosis in patients treated with gamma interferon, Lancet, 1, 893-895, 1987.

Phillips, M. J., Needham, M., and Weller, R. O.: Role of cervical lymph nodes in autoimmune encephalomyelitis in the Lewis rat, J. Pathol., 182, 457-464, 1997.

Picus, J., Aldrich, W. R., and Letvin, N. L.: A naturally occurring bone-marrow-chimeric primate. I. Integrity of its immune system, Transplantation, 39, 297-303, 1985.

Pietra, G., Romagnani, C., Mazzarino, P., Falco, M., Millo, E., Moretta, A., Moretta, L., and Mingari, M. C.: HLA-Erestricted recognition of cytomegalovirus-derived peptides by human CD8 + cytolytic $T$ lymphocytes, P. Natl. Acad. Sci. USA, 100, 10896-10901, 2003.

Pomeroy, I. M., Matthews, P. M., Frank, J. A., Jordan, E. K., and Esiri, M. M.: Demyelinated neocortical lesions in marmoset autoimmune encephalomyelitis mimic those in multiple sclerosis, Brain, 128, 2713-2721, 2005.

Pomeroy, I. M., Jordan, E. K., Frank, J. A., Matthews, P. M., and Esiri, M. M.: Diffuse cortical atrophy in a marmoset model of multiple sclerosis, Neurosci. Lett., 437, 121-124, 2008.

Richez, C., Truchetet, M. E., Schaeverbeke, T., and Bannwarth, B.: Atacicept as an investigated therapy for rheumatoid arthritis, Exp. Opin. Invest. Drugs, 23, 1285-1294, 2014.

Rodriguez, M. and Scheithauer, B.: Ultrastructure of multiple sclerosis, Ultrastruct. Pathol., 18, 3-13, 1994.

Saab, A. S., Tzvetanova, I. D., and Nave, K. A.: The role of myelin and oligodendrocytes in axonal energy metabolism, Curr. Opin. Neurobiol., 23, 1065-1072, 2013.

Samraj, A. N., Laubli, H., Varki, N., and Varki, A.: Involvement of a non-human sialic Acid in human cancer, Front. Oncol., 4, 1-13, 2014.

Sanvito, L., Constantinescu, C. S., Gran, B., and 't Hart, B. A.: The multifaceted role of interferon- $\gamma$ in central nervous system autoimmune demyelination, Open Autoim. J., 2, 151-159, 2010.
Sawcer, S., Hellenthal, G., Pirinen, M., et al.: Genetic risk and a primary role for cell-mediated immune mechanisms in multiple sclerosis, Nature, 476, 214-219, 2011.

Schnaar, R. L.: Brain gangliosides in axon-myelin stability and axon regeneration, FEBS Lett., 584, 1741-1747, 2010.

Schnaar, R. L., Gerardy-Schahn, R., and Hildebrandt, H.: Sialic acids in the brain: gangliosides and polysialic acid in nervous system development, stability, disease, and regeneration, Physiolog. Rev., 94, 461-518, 2014.

Segal, B. M., Constantinescu, C. S., Raychaudhuri, A., Kim, L., Fidelus-Gort, R., and Kasper, L. H.: Repeated subcutaneous injections of IL12/23 p40 neutralising antibody, ustekinumab, in patients with relapsing-remitting multiple sclerosis: a phase II, double-blind, placebo-controlled, randomised, doseranging study, Lancet Neurol., 7, 796-804, 2008.

Simons, M., Misgeld, T., and Kerschensteiner, M.: A unified cell biological perspective on axon-myelin injury, J. Cell Biol., 206, 335-345, 2014.

Singh, S., Metz, I., Amor, S., van der Valk, P., Stadelmann, C., and Bruck, W.: Microglial nodules in early multiple sclerosis white matter are associated with degenerating axons, Acta Neuropathol., 125, 595-608, doi:10.1007/s00401-013-1082-0, 2013.

Smith, P. A., Heijmans, N., Ouwerling, B., Breij, E. C., Evans, N., van Noort, J. M., Plomp, A. C., Delarasse, C., t Hart, B., Pham-Dinh, D., and Amor, S.: Native myelin oligodendrocyte glycoprotein promotes severe chronic neurological disease and demyelination in Biozzi ABH mice, Eur. J. Immunol., 35, 13111319, 2005.

Springer, S. A., Diaz, S. L., and Gagneux, P.: Parallel evolution of a self-signal: humans and new world monkeys independently lost the cell surface sugar Neu5Gc, Immunogenetics, 66, 671-674, 2014.

Stys, P. K., Zamponi, G. W., van Minnen, J., and Geurts, J. J.: Will the real multiple sclerosis please stand up?, Nat. Rev. Neurosci., 13, 507-514, 2012.

Taylor, R. E., Gregg, C. J., Padler-Karavani, V., Ghaderi, D., Yu, H., Huang, S., Sorensen, R. U., Chen, X., Inostroza, J., Nizet, V., and Varki, A.: Novel mechanism for the generation of human xeno-autoantibodies against the nonhuman sialic acid $\mathrm{N}$ glycolylneuraminic acid, J. Exp. Med., 207, 1637-1646, 2010.

't Hart, B. A.: Why does multiple sclerosis only affect human primates?, Mult. Scler. J., 1352458515591862, 2015.

't Hart, B. A., Bauer, J., Muller, H. J., Melchers, B., Nicolay, K., Brok, H., Bontrop, R. E., Lassmann, H., and Massacesi, L.: Histopathological characterization of magnetic resonance imaging - detectable brain white matter lesions in a primate model of multiple sclerosis: a correlative study in the experimental autoimmune encephalomyelitis model in common marmosets (Callithrix jacchus), Am. J. Pathol., 153, 649-663, 1998.

't Hart, B. A., Laman, J. D., Bauer, J., Blezer, E. D., van Kooyk, Y., and Hintzen, R. Q.: Modelling of multiple sclerosis: lessons learned in a non-human primate, Lancet Neurol., 3, 589-597, 2004a.

't Hart, B. A., Vogels, J. T., Bauer, J., Brok, H. P. M., and Blezer, E.: Non-invasive measurement of brain damage in a primate model of multiple sclerosis, Trends Mol. Med., 10, 85-91, 2004b.

't Hart, B. A., Brok, H. P., Remarque, E., Benson, J., Treacy, G., Amor, S., Hintzen, R. Q., Laman, J. D., Bauer, J., and Blezer, E. L.: Suppression of ongoing disease in a nonhuman primate model 
of multiple sclerosis by a human-anti-human IL-12p40 antibody, J. Immunol., 175, 4761-4768, 2005.

't Hart, B. A., Smith, P., Amor, S., Strijkers, G. J., and Blezer, E. L.: MRI-guided immunotherapy development for multiple sclerosis in a primate, Drug Discov. Today, 11, 58-66, 2006.

't Hart, B. A., Hintzen, R. Q., and Laman, J. D.: Multiple sclerosis - a response-to-damage model, Trends Mol. Med., 15, 235-244, 2009.

't Hart, B. A., Gran, B., and Weissert, R.: EAE: imperfect but useful models of multiple sclerosis, Trends Mol. Med., 17, 119-125, 2011.

't Hart, B. A., Jagessar, S. A., Haanstra, K., Verschoor, E., Laman, J. D., and Kap, Y. S.: The Primate EAE Model Points at EBV-Infected $B$ Cells as a Preferential Therapy Target in Multiple Sclerosis, Front. Immunol., 4, 145, doi:10.3389/fimmu.2013.00145, 2013.

't Hart, B. A., van Kooyk, Y., Geurts, J. J. G., and Gran, B.: The primate autoimmune encephalomyelitis model; a bridge between mouse and man, Ann. Clin. Transl. Neurol., 2, 581-593, 2015.

van der Valk, P. and Amor, S.: Preactive lesions in multiple sclerosis, Curr. Opin. Neurol., 22, 207-213, 2009.

van Kooten, C. and Banchereau, J.: Functions of CD40 on B cells, dendritic cells and other cells, Curr. Opin. Immunol., 9, 330-337, 1997.

van Oosten, B. W., Lai, M., Hodgkinson, S., Barkhof, F., Miller, D. H., Moseley, I. F., Thompson, A. J., Rudge, P., McDougall, A., McLeod, J. G., Ader, H. J., and Polman, C. H.: Treatment of multiple sclerosis with the monoclonal anti-CD4 antibody cMT412: results of a randomized, double-blind, placebo-controlled, MR-monitored phase II trial, Neurology, 49, 351-357, 1997.

van Zwam, M., Huizinga, R., Melief, M. J., Wierenga-Wolf, A. F., van Meurs, M., Voerman, J. S., Biber, K. P., Boddeke, H. W., Hopken, U. E., Meisel, C., Meisel, A., Bechmann, I., Hintzen, R. Q., t Hart, B. A., Amor, S., Laman, J. D., and Boven, L. A.: Brain antigens in functionally distinct antigen-presenting cell populations in cervical lymph nodes in MS and EAE. J. Mol. Med., 87, 273-286, 2009a. van Zwam, M., Huizinga, R., Heijmans, N., van Meurs, M., Wierenga-Wolf, A. F., Melief, M. J., Hintzen, R. Q., t Hart, B. A., Amor, S., Boven, L. A., and Laman, J. D.: Surgical excision of CNS-draining lymph nodes reduces relapse severity in chronic-relapsing experimental autoimmune encephalomyelitis, J. Pathol., 217, 543-551, 2009b.

Varki, A.: Loss of N-glycolylneuraminic acid in humans: Mechanisms, consequences, and implications for hominid evolution, Am. J. Phys. Anthropol., 33, 54-69, 2001.

Vierboom, M. P., Breedveld, E., Kondova, I., and t Hart, B. A.: Collagen-induced arthritis in common marmosets: a new nonhuman primate model for chronic arthritis, Arthritis Res. Ther., 12, R200, 2010.

Wang, B.: Sialic acid is an essential nutrient for brain development and cognition, Ann. Rev. Nutr., 29, 177-222, 2009.

Wilkin, T.: Autoimmunity: attack, or defence? (The case for a primary lesion theory), Autoimmunity, 3, 57-73, 1989.

Yamamura, T. and Miyake, S.: Diet, Gut Flora, and Multiple Sclerosis: Current Research and Future Perspectives, in: Multiple Sclerosis Immunology, edited by: Yamamura, T. and Gran, B., Springer Science + Business Media, New York, 115-126, 2013.

Zaguia, F., Saikali, P., Ludwin, S., Newcombe, J., Beauseigle, D., McCrea, E., Duquette, P., Prat, A., Antel, J. P., and Arbour, N.: Cytotoxic NKG2C + CD4 T cells target oligodendrocytes in multiple sclerosis, J. Immunol., 190, 2510-2518, 2013. 